\section{Comment on "Scaling of the Interface Roughness in Fe-Cr Superlattices: Self-Affine versus Non-Self-Affine"}

In a recent Letter, Santamaria et al. presented the first clear evidence for anomalous scaling of the interface roughness in sputtered metal-metal superlattices [1]. They observed that the scaling exponents did not fit in any of the existing model classes of surface growth that are known to exhibit anomalous scaling, and attributed the discrepancy to kinetic roughening being more complex in superlattices than in single films. Here we show that this explanation is unconvincing, since single films also exhibit anomalous scaling incompatible with the models quoted in [1], and qualitatively similar to that observed for superlattices.

Figure 1 shows the root-mean-square surface width $\sigma$ averaged over squares of side $l$, for $\mathrm{Cu}$ films prepared by electrodeposition from an alkaline $0.18 \mathrm{M} \mathrm{CuSO}_{4} / 0.25 \mathrm{M} \mathrm{K}_{4} \mathrm{P}_{2} \mathrm{O}_{7}$ electrolyte on $\mathrm{Au}(25 \mathrm{~nm}) / \mathrm{Ti}(5 \mathrm{~nm}) /$ glass substrates, and measured by atomic force microscopy. Note that the position of the crossover between power-law behavior $\left(\sigma \propto l^{\alpha_{\mathrm{loc}}}\right)$ and saturation $\left(\sigma=\sigma_{\text {sat }}\right)$, which is a measure of the lateral correlation length $l_{c}$, remains nearly constant as the deposition time, and correspondingly the film thickness increases. This indicates that the long and short length-scale dynamic exponents $\beta$ and $\beta_{\text {loc }}$ are approximately equal, which is exactly the behavior noted by Santamaria et al. [compare Fig. 1(b) of [1]] for columnar superlattices deposited at low-pressure (4-6 mTorr), although the value of $\beta$ is considerably larger in the electrodeposition case $(\beta=0.78 \pm 0.02)$.

Furthermore, electrodeposited $\mathrm{Cu}$ films can also show very similar scaling behavior to that determined for $\mathrm{Fe}-\mathrm{Cr}$ superlattices deposited at high pressure (8-10 mTorr) [1]. Values of $\alpha_{\mathrm{loc}}=0.75 \pm 0.05, \beta_{\mathrm{loc}}=$ $0.22 \pm 0.05$, and $\beta=0.76 \pm 0.05$ were reported for the superlattices, while values of $\alpha_{\mathrm{loc}}=0.78 \pm 0.05, \beta_{\mathrm{loc}}=$ $0.21 \pm 0.03$, and $\beta=0.57 \pm 0.05$ have been previously reported for $\mathrm{Cu}$ films electrodeposited from an organic additive-free acid $\mathrm{CuSO}_{4}$ electrolyte [2].

Although the two sets of $\mathrm{Cu}$ films were both grown on the same substrates, those deposited from the acid electrolyte that show scaling similar to the $\mathrm{Fe}-\mathrm{Cr}$ superlattices grown at high pressure were nanocrystalline, while those deposited from the alkaline electrolyte that show scaling qualitatively similar to the $\mathrm{Fe}-\mathrm{Cr}$ superlattices grown at low pressure were, similar to the super-

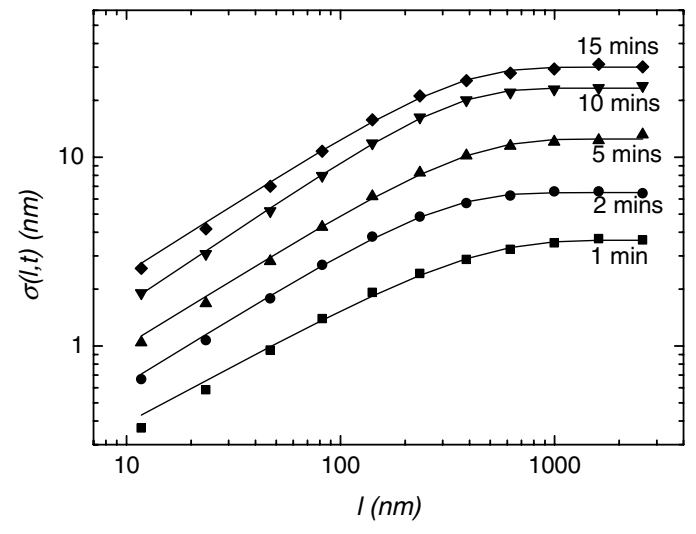

FIG. 1. $\quad \sigma(l, t)$ (root-mean-square surface width) measured using an atomic force microscope over regions of size $l$ for a series of electrodeposited films prepared from an alkaline $0.18 \mathrm{M} \mathrm{CuSO}_{4} / 0.25 \mathrm{M} \mathrm{K}_{4} \mathrm{P}_{2} \mathrm{O}_{7}$ electrolyte on $\mathrm{Au}(25 \mathrm{~nm}) / \mathrm{Ti}(5 \mathrm{~nm}) /$ glass substrates at current density $2.4 \mathrm{~mA} \mathrm{~cm}^{-2}$. Both axes are logarithmic. Each symbol corresponds to a film electrodeposited for a different time $t$ (written on the figure). The solid lines are fits to the data using the equation $\sigma=\sigma_{\text {sat }}\left\{1-\exp \left[-\left(l / l_{c}\right)^{2 \alpha_{\mathrm{loc}}}\right]\right\}^{1 / 2}$.

lattices, columnar. Clearly, kinetic roughening can be more complex in superlattices than in single films but, given the similarities between the experimental scaling data for electrodeposited single films and sputtered superlattices with similar microstructures, we suggest a more important reason why both may disagree with model predictions: The latter do not take microstructure, in particular, grain formation, into account.

W. Schwarzacher* and S. Huo ${ }^{\dagger}$

H. H. Wills Physics Laboratory

Tyndall Avenue

Bristol BS8 1TL, United Kingdom

Received 17 December 2002; published 12 September 2003 DOI: 10.1103/PhysRevLett.91.119601

PACS numbers: 68.55.Jk, 05.40.-a, 81.10.Aj, 81.15.Cd

*Electronic address: w.schwarzacher@bristol.ac.uk †Present address: Seagate Technology, 1 Disk Drive, Springtown, Northern Ireland.

[1] J. Santamaria, M. E. Gómez, J. L. Vicent, K. M. Krishnan, and I. K. Schuller, Phys. Rev. Lett. 89, 190601 (2002).

[2] S. Huo and W. Schwarzacher, Phys. Rev. Lett. 86, 256 (2001). 